\title{
Isolated oculomotor nerve palsy from minor head trauma
}

\section{C-C Chen, Y-M Pai, R-F Wang, T-L Wang, C-F Chong}

Br J Sports Med 2005;39:e34 (http://www.bjsportmed.com/cgi/content/full/39/8/e34). doi: 10.1136/bjsm.2004.016311

Isolated third cranial nerve palsies in head trauma patients can be the result of direct or indirect damage to the oculomotor nerve. They are usually associated with severe head trauma. We reported a case of isolated oculomotor nerve palsy associated with minor head injury. No initial loss of consciousness was recalled. Computed tomography (CT), magnetic resonance imaging (MRI), and magnetic resonance angiography (MRA) of the brain were normal. Previous reports in the literature were reviewed and the possible mechanism of injury was discussed. Head injuries are commonly seen in sports settings. Our case illustrated that even minor head trauma can cause isolated oculomotor nerve palsy in the absence of abnormal brain imaging findings.

$\mathrm{H}$ ead injuries are commonly seen in sports settings. Isolated third cranial nerve palsies are usually associated with severe head trauma as a result of direct or indirect damage to the oculomotor nerve. We report a case of isolated oculomotor nerve palsy associated with minor head injury.

\section{CASE REPORT}

A previously healthy 42 year old female suffered from double vision (diplopia) after sustaining a minor head injury that occurred when she lost control of her motorcycle on a curve. During the accident, she was wearing an ECE 2205-approved half-face helmet. She denied initial loss of consciousness and remembered that she sustained a direct impact to the right side of her head when she fell off her bike. She recalled no hyperflexion or hyperextension of her neck during the event. Past medical history revealed no diabetes, hypertension, or hyperlipidaemia. She had no previous episodes of head injuries or concussion and denied any history of smoking, habitual drinking, or drug abuse. She was $159 \mathrm{~cm}$ in height and $58 \mathrm{~kg}$ in weight. On examination, her vital signs were normal. She had normal cognition without retrograde amnesia. Examination of her right eye showed ptosis, lateral deviation of the eyeball, and a dilated, non-reactive pupil measuring $6 \mathrm{~mm}$ (fig 1). Ophthalmologic tests of vision and intraocular pressure were within normal ranges. The left eye was normal. There was a $2 \mathrm{~cm}$ laceration wound on the right temporal area of her head and some abrasions over her right elbow. Her neck and back showed no tenderness or deformity. Further physical and neurological examinations were normal. Radiographs of her skull, maxillae, cervical spine, and right elbow were normal. CT, MRI, and MRA of her head were also reported as normal. After discussion with a neurologist and an ophthalmologist, she was discharged with clear instructions and was followed up in the outpatient department. Four months after the injury, disabling diplopia and pupillary dilatation of her right eye persisted, although partial recovery of ptosis was found. She is currently using an
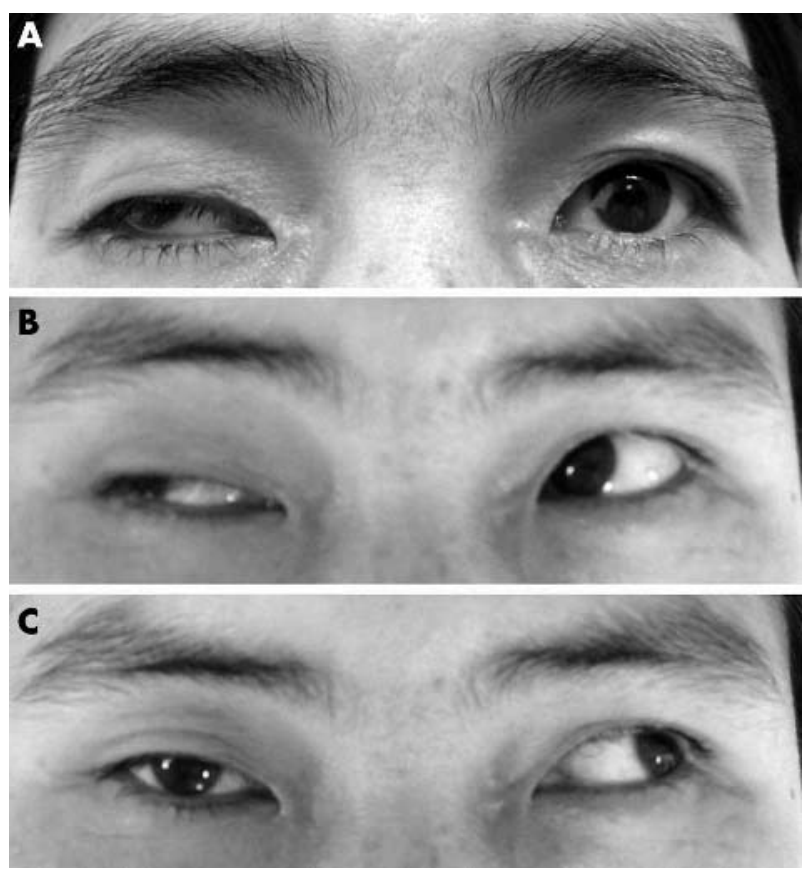

Figure 1 Photograph of the patient's extraocular movements on forward gaze (A), right gaze (B), and left gaze (C). (Photographs reproduced with permission)

occluding cosmetic contact lens in the right eye and is being followed up regularly in ophthalmology clinics.

\section{DISCUSSION}

The oculomotor nerve innervates the following extraocular muscles of either eye: superior rectus, inferior rectus, medial rectus, inferior oblique, levator palpebrae, ciliary muscle, and iris sphincter. With unilateral oculomotor nerve palsy, there will be ptosis with the involved eyeball usually infraducted and abducted. In addition, pupillary dilatation can cause symptomatic glare in bright light and paralysis of accommodation causes blurred vision for near objects. Isolated oculomotor nerve palsy due to closed head trauma is uncommon, with incidence ranging from 0 to $15 \% .^{1-5}$ All these patients sustained moderate to severe head injury with or without significant loss of consciousness. Elston studied 20 patients with traumatic third nerve palsy and concluded that patients who have closed head trauma severe enough to cause direct oculomotor nerve palsy usually have multiple permanent neurological deficits. ${ }^{5}$ Unusually, the isolated oculomotor nerve palsy in our patient occurred as a result of minor head injury. Two similar cases were previously reported to have oculomotor nerve palsy in the setting of minor head trauma. ${ }^{67}$ Both cases had normal brain CT scans.

Abbreviations: $\mathrm{CT}$, computed tomography; MRA, magnetic resonance angiography; $M R I$, magnetic resonance imaging 


\section{What is already known on this topic}

Isolated third cranial nerve palsy is usually seen in patients with severe head trauma who have abnormal brain CT, MRI, or MRA.

\section{What this study adds}

Isolated third cranial nerve palsy can occur in patients with minor head trauma who have negative findings on brain CT, MRI, and MRA. The actual mechanisms of damage may be direct or indirect.

In addition, the patient described by Muthu and Pritty showed negative findings on brain MRI and MRA.

The mechanisms of third cranial nerve injury can be direct or indirect. In patients with negative brain imaging studies (for example MRI and MRA), direct oculomotor nerve injury may be the result of extreme distraction of the nerve, rootlet avulsion, distal fascicular damage, or defective blood supply. On the other hand, indirect injury can occur as a result of compression, displacement, or deformity of the oculomotor nerve by space occupying lesions such as a cerebral aneurysm or an expanding haematoma. ${ }^{78}$ Physicians must be alert to any elements in the history or physical examination that point towards such serious neurosurgical conditions and make the appropriate referrals in a timely way. The actual mechanisms of damage to the oculomotor nerve from minor head trauma are not apparent. Nagaseki $e t a l^{6}$ concluded that the ophthalmoplegia was due to downward displacement of the brainstem at the time of impact which directly injured the pupillomotor fibres on the ventromedial surface of the third nerve at the posterior petroclinoid ligament. In addition to mechanical injuries such as rootlet avulsion and distal fascicular damage, Muthu and Pritty ${ }^{7}$ suggested that the cranial nerves may suffer from disturbances in blood supply or detrimental biochemical effects arising from head injury.

The prognosis of traumatic oculomotor palsy is poor and full recovery is uncommon. ${ }^{378}$ A prolonged period (up to years) of healing process was usually anticipated. Although definitive surgery was not available, ${ }^{9}$ occluding spectacle lenses are helpful for patients awaiting recovery and botulinum toxin injection has been useful in treating exotropia of oculomotor nerve palsy in certain patients. ${ }^{10}$

In conclusion, our case illustrated that minor head trauma can cause isolated oculomotor nerve palsy in the absence of abnormal findings on brain CT, MRI, and MRA. Although rare, this disease entity should be kept in mind in the management of all patients who sustain head trauma regardless of its severity.

\section{Authors' affiliations}

C-C Chen, Y-M Pai, R-F Wang, T-L Wang, Shin Kong Wu Ho-Su Memorial Hospital, Taipei, Taiwan

C-F Chong, School of Medicine, Fu-Jen Catholic University, Taipei, Taiwan

Competing interests: none declared

The patient detailed in this case report agreed to her details being published.

Correspondence to: Chee-Fah Chong, Shin Kong Wu Ho-Su Memorial Hospital, Emergency Department, Taipei 111, Taiwan; jack.cfchong@ msa.hinet.net

Accepted 11 January 2005

\section{REFERENCES}

1 Tiffin PAC, MacEwen CJ, Craig EA, et al. Acquired palsy of the oculomotor, trochlear and abducens nerves. Eye 1996;10:377-84.

2 Solomons NB, Solomon DJ, DeVilliers JC. Direct traumatic third nerve palsy. $S$ Afr Med J 1980;58:109-111.

3 Memon MY, Paine KWE. Direct injury of the oculomotor nerve in craniocerebral trauma. J Neurosurg 1971;35:461-4.

4 Eyster EF, Hoyt WF, Wilson CB. Oculomotor palsy from minor head trauma. JAMA 1972;220:1083-6

5 Elston JS. Traumatic third nerve palsy. Br J Ophthalmol 1984;68:538-43.

6 Nagaseki Y, Shimizu T, Kakizawa T, et al. Primary internal ophthalmoplegia due to head injury. Acta Neurochir 1989;97:117-22.

7 Muthu P, Pritty P. Mild head injury with isolated third nerve palsy. Emerg Med J 2001;18:310-1.

8 Heinz J. Cranial nerve avulsion and other neural injuries. Med J Aust 1969;2:1246-9.

9 Miller NR. The oculomotor nerves. Curr Opin Neurol 1996;9:21-5.

10 Saad N. Lee J. The role of botulinum toxin in third nerve palsy. Aust N Z J Ophthalmol 1992;20:121-7. 